\title{
Rursus
}

r.muksus

Poiétique, réception et réécriture des textes antiques

2| 2007

Le modèle animal (II)

\section{Intelligence et vertus animales : Montaigne lecteur de la zoologie antique}

\section{Thierry Gontier}

\section{(2) OpenEdition \\ 1 Journals}

Édition électronique

URL : http://journals.openedition.org/rursus/115

DOI : $10.4000 /$ rursus. 115

ISSN : 1951-669X

Éditeur

Université Nice-Sophia Antipolis

Référence électronique

Thierry Gontier, « Intelligence et vertus animales : Montaigne lecteur de la zoologie antique », Rursus [En ligne], 2 | 2007, mis en ligne le 04 mai 2007, consulté le 10 décembre 2020. URL : http:// journals.openedition.org/rursus/115; DOI : https://doi.org/10.4000/rursus.115

Ce document a été généré automatiquement le 10 décembre 2020.

Rursus 


\title{
Intelligence et vertus animales : Montaigne lecteur de la zoologie antique
}

\author{
Thierry Gontier
}

1 De très nombreuses références aux animaux parsèment les Essais de Montaigne. Il s'agit tantôt de simples allusions, tantôt de développements assez amples. Le plus significatif, et celui qui a fait passer Montaigne à la postérité comme l'initiateur d'un genre littéraire nouveau empruntant à la fois à la littérature paradoxale et à la philosophie morale, est sans doute la longue plaidoirie en faveur des animaux qui occupe, dans l'édition de Pierre Villey de l'Essai II, 12, l'« Apologie de Raimond Sebond», plus de 30 pages - soit la taille d'un bel essai.

Tout, ou presque, dans cette plaidoirie, provient d'un fonds antique.

3 Tout d'abord les exemples. Les 30 pages dont nous parlons en comprennent plus de 130 . Si Montaigne fait des références ponctuelles aux recueils renaissants de mirabilia (tels ceux d'Agrippa, Gomara, Bodin, Bruès, Boaystuau, etc.), ses sources principales sont antiques. On trouve des emprunts à Pline, Élien, Lucien, Aulu-Gelle, etc. La première de toutes les références est cependant constituée par la seconde partie du traité de Plutarque qui porte pour titre (dans l'édition de Jacques Amyot que Montaigne utilise) Quels animaux sont les plus avisés, ceux de la terre ou ceux de l'eau ? ${ }^{1}$, dont Montaigne reprend la quasi-totalité des anecdotes zoologiques, au point que l'on peut parler d'une véritable réécriture du texte.

Outre les exemples, Montaigne reprend à la littérature antique sur les animaux le plan de son propre développement, qui suit jusqu'à un certain point (car Montaigne fait de nombreuses digressions, dont certaines très longues) la division traditionnelle entre la raison proférée (logos prophorikos) et la raison intérieure (logos endiathètos). Cette distinction apparait chez Platon et chez Aristote, mais c'est dans la philosophie expressive des stoïciens qu'elle revêt une fonction axiomatique et quasi métaphysique, avant de devenir un simple topos autour duquel s'organisent les arguments des différents adversaires dans la querelle entre stoïciens et académiciens sur l'intelligence des animaux. Montaigne a pu prendre connaissance de cet ordre d'exposition chez Plutarque 
(Quels animaux sont les plus avisés? et, dans une moindre mesure, Si les animaux usent de raison), chez Sextus Empiricus (l'éloge du chien en Hypotyposes pyrrhoniennes, I, 14, dont Montaigne connaît la traduction latine d'Henri Estienne de 1562), ainsi que, peut-être, chez Porphyre (troisième livre du De abstinentia). On ne saurait par ailleurs limiter cette querelle au simple cadre de la polémique entre le Portique et la Nouvelle Académie, puisque Sextus Empiricus se réclame du pyrrhonisme (et Porphyre du végétarisme); à l'inverse, la position stoïcienne sur les animaux a été aussi soutenue par les tenants de l'anthropocentrisme judéo-chrétien, comme Philon d'Alexandrie ${ }^{2}$ et Origène (quatrième livre du Contre (else). Bref, il s'agit d'une dispute assez générale dont les enjeux sont le dogmatisme et l'anthropocentrisme. Chez Montaigne aussi, on trouve une courte partie (p. 452-454 de l'édition de Pierre Villey) sur la raison proférée (le langage des animaux) suivie d'une bien plus longue (p. 454-482) sur la raison intérieure (les autres signes de l'intelligence des animaux). Le plan d'exposition de Montaigne épouse ce schéma jusque dans ses ramifications : l'argumentation sur la raison intérieure des animaux se divisait dans le débat antique en un développement sur les facultés industrieuses des animaux et une autre sur leurs qualités morales (les différentes vertus et les vices des animaux) - ces divisions sont toutes reprises par Montaigne ${ }^{3}$. Il reste que les exemples de Plutarque sont chez lui totalement réorganisés et re-contextualisés selon une perspective qui lui est absolument propre. Il en va de même pour le plan d'ensemble, qui n'a plus rien d'une simple trame scolaire permettant de ranger arguments et exemples : elle prend, dans l' Apologie de Raimond Sebond, véritablement vieen se mettant au service d'une stratégie d'argumentation complexe et originale.

Plus généralement, Montaigne ne se contente pas de recopier les Antiques. Nous voulons ici tenter d'éclairer dans quelques grandes lignes ce travail montaigniste de réappropriation et de translatio opérées sur la littérature zoologique des Anciens.

\section{Acatalepsie et critique de l'anthropocentrisme : Montaigne et Sextus Empiricus}

6 L'éloge des animaux forme le premier volet d'un long exposé sceptique. Cet exposé sceptique, constitue le vrai objet de l'«Apologie de Raimond Sebond", le propos apologétique annoncé par le titre se trouvant vite comme «débordé » l'ampleur de l'appareil sceptique que Montaigne met en œuvre. L'utilisation de la défense des animaux au sein d'une argumentation sceptique est une constante de la littérature philosophique de l'Antiquité. Les Académiciens associaient l'éloge des animaux à un discrédit général de la phantasia kataleptikè (la représentation compréhensive - c'est-à-dire auto-certifiée et entrânant irrésistiblement l'assentiment) des Stoïciens. Par son sensualisme et par son immanentisme radical, le stoïcisme se présente en effet comme l'envers du platonisme : toute la théorie stoïcienne de la connaissance se fonde en dernier recours sur la certitude inébranlable de l'impression sensible. La "Nouvelle Académie » c'est-à-dire (selon les doxographies de Cicéron et de Sextus) l'Académie d'Arcesilias et de Carnéade, représente une réaction du platonisme contre ce sensualisme et son affirmation de l'immanence du critère de la vérité (le fameux métron platonicien) au monde sensible qui en résulte. Pour sauver la transcendance du métron, les Académiciens ont été conduits à combattre la certitude sensible des Stoïciens. Il est vrai que la Nouvelle Académie a été conduite aussi bien à combattre la certitude " tout court ", mettant pour le coup en péril les fondements mêmes du platonisme : ainsi pour Arcésilias, qui, selon Cicéron, niait «negabat quod esse 
quidquam quod sciri potest » (niait que quoi que ce soit puisse être connu) ${ }^{4}$. La stratégie d'ensemble reste cependant orientée vers le discrédit de la connaissance sensible. Le meilleur témoin de cette position anti-stoïcienne est Cicéron, dont les Académiques présentent une défense de l'Académie de Carnéade et de Philon de Larissa contre le retour d'Antiochus d'Ascalon à une forme de dogmatisme stoïcisant. On ne trouve malheureusement chez Cicéron qu'une mince allusion à la question de l'animal. Elle est cependant significative par le contexte dans lequel cette allusion apparait :

A supposer que le sens de la vue soit véridique, jusqu'à quelle distance voyonsnous? Je vois d'ici la villa de Catulus à Cumes [la scène a lieu dans la villa d'Hortensius près de Baules - dont à côté de Cumes], je vois bien la région où se trouve la villa de Pompéi, mais cette villa elle-même, je ne la vois pas; non qu'il y ait quelque obstacle qui s'y oppose [les Stoïciens concédaient que les sens puissent être ainsi empêchés], mais mon regard ne peut s'étendre assez loin [...] On parle dans les écoles d'un homme qui voyait un objet distant de dix-huit cent stades. Et les oiseaux voient encore plus loin. Je répondrai donc avec audace à votre dieu [qui est censé nous avoir donné des sens aptes à fonder la certitude de toute connaissance qui s'appuie sur leur bon usage] que je ne suis pas du tout content des yeux qu'il m'a donnés (me plane his oculis non esse contentum) ${ }^{5}$.

7 L'animal me fournit une expérience indirecte de la relativité, voire de l'imperfection, de mes sensations; de sorte qu'il m'est impossible de faire d'eux le critère de la certitude, et par là d'élaborer sur leur fondement quelque vérité que ce soit. Cet argument de ce que l'on pourrait nommer «l'animal mesure » se trouvait déjà dans le Théétète de Platon, lorsque Socrate répondait à Protagoras et ses alliés sensualistes et phénoménistes qu'il était impossible de soutenir que « telle t'apparaît chaque couleur, telle aussi elle apparait à un chien ou à tout autre animal ${ }^{6}$. Dans ce cas, rien ne permet plus de privilégier les sensations humaines et il est aussi possible de dire que «la mesure de toutes choses ( pantôn khrèmatôn métron), c'est le pourceau ou le babouin ou quelque bête encore plus bizarre (atopôtéron) parmi celles qui ont sensation " ?

8 C'est chez Sextus Empiricus que cet argument se trouve pleinement développé. Sextus Empiricus est l'élève d'Enésidème, qui reprend la critique cicéronienne de la dérive dogmatique de la Nouvelle Académie (celle d'Antiochus): quoique se réclamant du pyrrhonisme (qui est à l'époque de Cicéron une doctrine désuète) contre la Nouvelle Académie, les parentés sont nombreuses entre la défense du pyrrhonisme de Sextus et la défense d'une Académie authentique (celle de Carnéade) de Cicéron. La "variété des animaux» (differentia animalium ${ }^{8}$ ) constitue le tout premier des tropes en faveur du scepticisme :

Si ergo diversae sunt phantasiae pro diversitate animalium, quas dijudicare est impossibile : assendum de extrinsecus subjectis sustinere necesse est ${ }^{9}$.

Nous traduisons ainsi :

Si les impressions sensibles diffèrent en fonction de la diversité des animaux, et qu'il est impossible de trancher entre elles, il est nécessaire de suspendre notre jugement sur les objets extérieurs.

10 Or il est facile d'inférer cette différence de celle qui existe dans la génération des animaux et dans leur constitution corporelle (ex diversis eorum generationibus, et differentia quae est in corporum constitutionibus) ${ }^{10}$. A ces considérations pour ainsi dire «techniques ", Sextus ajoute un éloge du chien (même exemple que dans le Théétète), qui, écrit-il, «l'emporte sur nous dans le registre de la sensation ", de par l'acuité de son odorat, de sa vue et de son audition. 
11 Le développement de Sextus fournit sans doute à Montaigne la trame première de son propre éloge des animaux selon les grandes divisions dont nous avons parlé. Ce qui est intéressant ici, c'est que Montaigne ne pose nullement cette question de la valeur de sensations animales. Il le fera, mais plus tard, dans la troisième partie de l'« Apologie » ${ }^{11}$, lorsqu'il s'attaquera à la connaissance à partir de ses tout premiers fondements :

Toute cognoissance s'achemine en nous par les sens : ce sont nos maistres [...]. La science commence par eux et se resout en eux [...]. Les sens sont le commencement et la fin de l'humaine cognoissance [...] Par leur voye et entremise s'achemine toute nostre instruction ${ }^{12}$.

Parmi les arguments évoqués par Montaigne pour jeter la suspicion sur les sens, nous lisons :

Si les sens sont noz premiers juges, ce ne sont pas les nostres qu'il faut seuls appeler au conseil, car en cette faculté les animaux ont autant ou plus de droit que nous. Il est certain qu'aucuns ont l'ouye plus aigue que l'homme, d'autres la veue, d'autres le sentiment [c'est-à-dire l'odorat] ${ }^{13}$

13 Les exemples que donnera Montaigne (tirés de Pline et Plutarque) seront bien plus parlants que la simple trame argumentative fournie par Sextus. Chez Montaigne comme chez Sextus, il ne s'agit nullement de détruire le sensualisme au sens d'une théorie qui fonde la certitude de la connaissance sur celle des sensations: bien au contraire, Montaigne s'appuie sur deux citations de Lucrèce pour montrer qu'il n'y a pas d'autre forme de connaissance que celle qui se fonde sur les sens. La stratégie montaigniste consiste bien plutôt à tirer parti du sensualisme pour démontrer la vanité de toute forme de connaissance. De ce fait, le scepticisme prend une forme radicale et universelle qu'il n'avait pas dans l'Académie. Ce thème de la relativité de la certitude sensible ne constitue par ailleurs chez Montaigne qu'un thème annexe. Montaigne est par exemple tout à fait étranger aux arguments techniques de Sextus sur le mode de génération des animaux et leur constitution corporelle - il se contente de prendre la différence des sensations comme un fait (ou au minimum une possibilité) qui n'a pas à être démontré. Mais surtout, ce thème de la valeur de la certitude sensible est totalement absent de l'éloge des animaux : nous sommes ici à un tout autre endroit de l'«Apologie», plus de cent pages plus loin. On conclura que la longue plaidoirie de Montaigne en faveur des animaux, tout en épousant les cadres formels de la querelle anti-stoïcienne, déborde le cadre argumentatif originaire, à savoir la réfutation du sensualisme stoïcien.

Cette plaidoirie retient pourtant de Sextus une structure argumentative générale - ce que nous pourrions nommer la structure duelle de l'argument des animaux. La conclusion relativiste dépend en effet chez Sextus Empiricus de deux prémisses distinctes: 1 / Affirmation de l'altérité animale : l'animal manifeste un autre mode de sensation que le nôtre, un mode dont la caractéristique principale est de nous être étranger; 2 / Affirmation de l'égalité des animaux et des hommes, ou, plus exactement de la noninfériorité des animaux vis-à-vis des hommes : il n'y a aucune raison de privilégier les sensations humaines vis-à-vis des sensations animales. La "différence » animale joue ainsi à la fois comme étrangeté et comme égalité dans la distinction: c'est ce couple étrangeté-égalité qui donne au thème de la «différence » animale sa force proprement sceptique.

On retrouve bien chez Montaigne, quoiqu'à une niveau bien plus général (non limité à la critique de la certitude des sens) ce double emploi de la figure animale : 
161 / L'animal constitue l'exemple même d'un être incompréhensible à l'homme. Ce qui est ici exploité de l'altérité animale, c'est son étrangeté. C'est là le sens du texte qui introduit la plaidoirie de l'« Apologie » :

Comment [l'homme] cognoit il, par l'effort de son intelligence, les branles internes et secrets des animaux? par quelle comparaison d'eux à nous conclud il la bestise qu'il leur attribue ? ${ }^{14}$

C'est aussi le sens du texte qui introduit le passage sur la religion des animaux :

Tout ce qui nous semble estrange, nous le condamnons, et ce que nous n'entendons pas [i.e. nous le condamnons aussi] : comme il advient au jugement que nous faisons des bestes. Elles ont plusieurs conditions qui se rapportent aux nostres : de celles-là par comparaison, nous pouvons tirer quelque conjecture; mais de ce qu'elles ont particulier, que sçavons nous que c'est ? ${ }^{15}$

« Nous ne pouvons prendre en aucune part ce qui nous est caché », dira un peu plus loin Montaigne: cette étrangeté renvoie cependant moins à un autre mode de sentir, de connaître ou d'agir (comme c'est le cas chez Sextus), qu'à un mode de connaissance et d'action qui nous est radicalement étranger et dont nous ne pouvons saisir le principe.

192 / L'animal constitue par ailleurs un prétendant aussi légitimé que l'homme à fournir la mesure des choses et à régenter l'être sous un mode herméneutique. La question est ici : " au fond, qu'est-ce qui permet à l'homme de s'ériger en mesure de certitude aux dépens des animaux ». C'était là le sens de l'éloge du chien chez Sextus. Le texte le plus parlant de Montaigne est ici la prosopopée de l'oison, qui se trouve d'ailleurs à un autre endroit de l' Apologie, lorsque Montaigne s'attaque aux théologies anthropomorphes:

Car pourquoy ne dira un oison ainsi: Toutes les pieces de l'univers me regardent ; la terre me sert à marcher, le Soleil à m'esclairer, les estoilles à m'inspirer leurs influances; j'ay telle commodité des vents, telle des eaux; il n'est rien que cette voute regarde si favorablement que moy; je suis le mignon de nature; est-ce pas l'homme qui me traite, qui me loge, qui me sert? c'est pour moy qu'il faict et semer et mouldre ; s'il me mange, aussi faict il bien l'homme son compaignon, et si fay-je moy les vers qui le tuent et qui le mangent ${ }^{16}$.

20 La « différence » animale ne joue plus ici en tant qu'étrangeté. L'argument ne fonctionne au contraire que parce qu'on reconnaît à l'animal une certaine égalité vis-à-vis de l'homme. La différence vaut ici pour égalité dans la distinction.

21 L'animal représente ainsi un double défi à notre raison. Pris comme un objet d'interprétation, il représente ce sur quoi butte notre dogmatisme herméneutique; pris comme un sujet d'interprétation aussi qualifié que nous, il représente ce sur quoi butte notre prétention à dominer le monde et à constituer la mesure exclusive de l'être. La complexité du texte de Montaigne tient pour une grande part à l'imbrication de ces deux motifs (un troisième motif, d'ordre moral, doit encore être ajouté : nous y reviendrons dans un instant). Le très fameux ajout de la couche $[C]$ « Quand je me jouë à ma chatte, qui sçait si elle passe son temps de moy plus que ne fay d'elle ${ }^{17}$, se situe par exemple à l'intersection des deux motifs, posant implicitement les deux questions : 1 / Qu'est-ce que je comprends de ma chatte ? 2/Qui de nous deux est le plus légitimement apte à se constituer en sujet capable d'interpréter notre action commune? Ainsi, à travers l'éloge des animaux, la prétention à la connaissance est attaquée tant du côté de l'objet (rien ne peut être connu) que du sujet (l'homme n'est pas habilité à connaitre l'essence des choses).

22 La différence principale entre le propos de Montaigne et celui de Sextus (ou plus généralement de l'anti-stoïcisme antique) tient au statut central qu'occupe chez 
Montaigne la critique de l'anthropocentrisme, soit d'un certain rapport de l'homme au monde et à l'être. Re-citons le texte qui introduit le développement sur les animaux de l' «Apologie », mais cette fois en le replaçant dans son contexte :

La presomption est nostre maladie naturelle et originelle. La plus calamiteuse et fraile de toutes les creatures, c'est l'homme, et quant et quant la plus orgueilleuse. Elle se sent et se void logée icy, parmy la bourbe et le fient du monde, attachée et clouée à la pire, plus morte et croupie partie de l'univers, au dernier estage du logis et le plus esloigné de la voute celeste, avec les animaux de la pire condition des trois ; et se va plantant par imagination au dessus du cercle de la Lune et ramenant le ciel soubs ses pieds. C'est par la vanité de cette mesme imagination qu'il s'egale à Dieu, qu'il s'attribue les conditions divines, qu'il se trie soy mesme et separe de la presse des autres creatures, taille les parts aux animaux ses confreres et compaignons, et leur distribue telle portion de facultez et de forces que bon luy semble. Comment cognoit il, par l'effort de son intelligence, les branles internes et secrets des animaux? par quelle comparaison d'eux à nous conclud il la bestise qu'il leur attribue ? ${ }^{18}$

Et Montaigne enchaîne directement sur la question de la communication entre l'homme et les animaux. On voit ici une différence essentielle entre Montaigne et ses sources antiques : là où Philon, Sextus, Plutarque, Origène et Porphyre reprenaient un schéma scolaire, Montaigne le fait vivre à l'intérieur d'une argumentation entièrement centrée sur l'attaque de l'anthropocentrisme herméneutique, déniant généralement à l'homme le droit d'interpréter le monde, et même lui déniant toute relation privilégiée à la vérité des êtres.

Cette critique de l'anthropocentrisme n'est certes pas absente de la perspective académicienne. Nous avons vu tant chez Platon (définissant ici la position relativiste et sensualiste) que chez Cicéron et chez Sextus qu'elle était ordonnée au discrédit jeté sur la certitude sensible. Les stoïciens en effet fondaient leur confiance absolue dans la certitude des sens sur une conception forte de la Providence divine, ayant doté l'homme des moyens nécessaires d'accès à l'être des choses (qui, pour les stoïciens, consiste en un certain degré de tension du pneuma ${ }^{19}$. Citrons à ce titre le début du discours de Lucullus (partisan ici du dogmatisme stoïcien) au second livre de la première version des Académiques de Cicéron:

Commençons par les sens : leurs jugements sont si clairs et si certains (clara et certa) que, si le choix était donné à notre nature et si un dieu lui demandait si elles se contente d'avoir des sens intacts et sains (integris incorruptisque) ou si elle réclame quelque chose de mieux, je ne vois pas ce qu'elle pourrait souhaiter de plus (non videam quid quaerat amplius) ${ }^{20}$.

Ce à quoi, nous l'avons vu, Cicéron répondait avec impertinence (audaciter) qu'il n'était pas satisfait des yeux que ce dieu lui avait donnés. Il s'agit ici de saper cette confiance anthropocentrique qui établirait les facultés cognitives de l'homme en critère de certitude. On peut dire en ce sens que Montaigne reprend cette critique d'un anthropocentrisme herméneutique (le droit de l'homme à fournir le sens de toute chose) en l'élargissant bien au-delà du thème initial de la certitude sensible. La critique de l'anthropocentrisme acquiert chez Montaigne un statut d'autonomie qu'elle n'avait pas dans la critique traditionnelle du stoïcisme. Cette critique se trouvera articulée directement à une perspective morale dont nous devrons définir le sens. 


\section{L'usage des mirabilia : Montaigne et Plutarque}

Le radicalisme du projet sceptique trouve un prolongement dans l'attitude de Montaigne face aux exemples de Plutarque. Dans la seconde partie du traité « Quels animaux sont les plus avisés?", les éloges des animaux terrestres et aquatiques sont principalement les sujets à des exercices rhétoriques: Plutarque, qu'il convient de définir comme un rationaliste prudent plus que comme un sceptique, ne s'identifie ni au chasseur, ni au pêcheur dans leur concours de vantardise. Il se situe à un autre niveau - celui d'Autobulus qui, dans la première partie du traité, prononce son jugement contre la chasse et la pêche. Les quelques critiques ironiques que Phédime (le pêcheur) adresse à Aristotime (le chasseur) montrent qu'il ne faut pas prendre pour argent comptant les mirabilia qu'il rapporte. Voici quelques échantillons de ces réserves :

Je ne vous alléguerai ni les opinions des Philosophes, ni les fables des Égyptiens, ni les contes des Indiens ou des Libyens, sans aucune preuve de témoins, mais vous réciterai et produirai choses toutes notoires, qui se peuvent voir à l'œil et partout ${ }^{21}$. Quant à l'histoire que tu as alléguée des éléphants, mon bel ami, qu'ils jettent dedans la fosse tout ce qu'ils peuvent fouiller et arracher, pour faire comme une levée à leur compagnon, tombé dedans, afin de l'aider à sortir, elle est merveilleusement étrange et de fort lointain pays: aussi nous commande-t-elle comme par édit royal, venant des livres du roi Juba, de la croire ${ }^{22}$.

Si tu voulais que l'on crût ton conte du chien d'Hésiode, il fallait pas que tu ne laissasses pas aussi derrière les Dauphins ${ }^{23}$.

Certes, les contes de Phédime ne valent pas mieux que ceux d'Aristotime. Celui-ci finit par le reconnaître lui-même à la fin de son discours :

Mais pour autant que je vous avais protesté au commencement que je ne vous alléguerais aucune fable, et néanmoins je ne sais comment sur le propos de ces Dauphins je suis allé donner jusqu'à Ulysse et à Cœranus, un peu trop loin de vérisimilitude [de la vérité], je me condamne moi-même à l'amende ${ }^{24}$

Montaigne, lui, reprend tous ces mirabilia, en faisant purement et simplement disparaître tout ce qui, dans le récit de Plutarque, pouvait introduire quelque distanciation, et en rapportant à l'autorité directe de Plutarque tous les contes qu'il s'était contenté de faire exposer par ses personnages. Il refuse même à plusieurs reprises les réserves prudentes que Plutarque ajoute au récit. Un exemple particulièrement parlant se trouve dans la reprise de l'histoire du "chien de Chrysippe", devant un carrefour à trois chemins, raisonnant par élimination pour savoir lequel il doit suivre afin de rejoindre sa proie. Cher Plutarque, Aristotime raconte l'histoire, mais se refuse finalement à croire qu'un raisonnement dialectique complexe, par élimination successive des possibilités, soit nécessaire pour expliquer le comportement du chien. La vivacité du sens suffit ici. Citons Plutarque :

Les Dialecticiens même tiennent que le chien, se trouvant en un carrefour divisé en plusieurs chemins, use de l'argumentation qui s'appelle l'énumération suffisante des parties et discourt ainsi en lui-même : il faut que la bête que je chasse, soit passée par l'un de ces chemins ; or, elle n'est allée ni par celui-ci, ni par celui-là, elle est donc passé par ce troisième. Le sentiment du nez ne lui donne en effet que les prémisses et le discours de la raison lui donne à entendre la nécessité de la conséquence et de la conclusion. Le chien n'a que faire de ce témoignage des Dialecticiens , car il est faux et mensonger, parce que c'est l'odorat et le sentiment du nez qui par la trace du pied et par la fluxion de l'odeur émanant de la bête lui montre par où 
elle a fui, sans se soucier des propositions divisées ou conjointes ni de la suffisante énumération des parties ${ }^{25}$. exigée - on peut sauver les phénomènes à moindre frais. Le chien continue à flairer sa piste - l'interprétation s'appuie sur un rapport faux des faits. Curieusement, Montaigne ne reprend pas ad litteram le texte de Plutarque comme il le fait ailleurs. Il combine ce texte à un autre, celui de Sextus Empiricus que voici :

Selon Chrysippe (secundum Chrisippum), qui pourtant est extrêmement hostile aux animaux sans raison (alogoi), le chien a même part à leur fameuse dialectique. Ainsi le philosophe précité dit que le chien a recours au cinquième indémontrable composé de plusieurs branches (tertii notitiam per multa anapodicta cinsequi [particeps est]), quand, étant arrivé à un carrefour de trois voies et ayant reconnu par son flair que le gibier n'a pas emprunté deux de ces voies, il se précipite immédiatement dans la troisième sans l'avoir flairée. En effet, dit cet auteur ancien, le chien fait en puissance le raisonnement suivant (ratiocinaretur hoc modo) : «Le gibier a pris soit celle-ci, soit celle-là, soit celle-là, or ce n'est ni celle-ci ni celle-là ; donc c'est cellelà » (aut hac, aut istac, aut illac transiit fera : neque autem hac, neque istac : ergo illac) ${ }^{26}$.

Voici maintenant, à titre de comparaison, le texte de Montaigne :

Chrysippus, bien que en toutes autres choses autant desdaigneux juge de la condition des animaux que nul autre philosophe, considerant les mouvements du chien qui, se rencontrant en un carrefour à trois chemins, ou à la queste de son maistre qu'il a esgaré, ou à la poursuitte de quelque proye qui fuit devant luy, va essayant l'un chemin apres l'autre, et, apres s'estre asseuré des deux et n'y avoir trouvé la trace de ce qu'il cherche, s'eslance dans le troisiesme sans marchander, il est contraint de confesser qu'en ce chien là un tel discours se passe : J'ay suivy jusques à ce carre-four mon maistre à la trace; il faut necessairement qu'il passe par l'un de ces trois chemins; ce n'est ny par cettuy-cy, ny par celuy-là ; il faut donc infailliblement qu'il passe par cet autre; et que, s'asseurant par cette conclusion et discours, il ne se sert plus de son sentiment au troisiesme chemin, ny ne le sonde plus, ains s'y laisse emporter par la force de la raison. Ce traict purement dialecticien et cet usage de propositions divisées et conjoinctes et de la suffisante enumeration des parties, vaut-il pas autant que le chien le sçache de soy que de Trapezonce [i.e. George de Trézibonde] ${ }^{27}$.

Montaigne reprend à Plutarque la définition du raisonnement du chien (la suffisante énumération des parties), mais il se réfère à Sextus lorsqu'il attribue cette analyse à Chrysippe (et non généralement au «Dialecticiens»). Il reprend surtout à Sextus l'absence de toute réserve quant à l'interprétation de l'action animale. Au fond, pour Montaigne, Plutarque n'a aucune raison de refuser l'interprétation qui est celle de Sextus, et qui conduit à placer l'animal à l'égal de l'homme. Et ce d'autant plus que Plutarque luimême admet, dans l'exemple du renard de Thrace posant son oreille sur la glace pour évaluer son épaisseur avant de s'aventurer dessus, que celui-ci procède par « une ratiocination et consequence tirée du sens naturel», et non par la simple vivacité du sens :

Car d'attribuer cela seulement à une vivacité du sens de l'ouye, sans discours et sans consequence, c'est une chimere, et ne peut entrer en nostre imagination ${ }^{28}$.

Ici, les différences entre Montaigne et Plutarque sont manifestes. Plutarque refuse un scepticisme systématique. Son attitude est encore une fois celle d'un rationaliste prudent, défendant ce qu'on pourrait appeler une philosophie du «bon sens" contre les excès dogmatiques de la théorie stoïcienne. Montaigne refuse quant à lui la discrimination herméneutique (qui fait que le renard de Thrace agit par raisonnement, mais non le chien 
de Chrysippe). Faute d'un critère rationnel, rien ne permet de critiquer les mirabilia, aussi incroyables nous paraissent-ils. Montaigne est crédule, si l'on veut - mais il convient de souligner qu'il s'agit ici d'une crédulité méthodique et pour ainsi dire construite ${ }^{29}$. Ce à quoi tend Montaigne, c'est à abolir toute frontière entre le normal et le merveilleux, à disséminer la nature en autant de cas singuliers (le chien de Chrysippe, le mulet de Thalès, le lion d'Andronicus, etc.) interdisant toute entreprise herméneutique de la part de l'homme et le privant de sa royauté imaginaire sur les êtres.

La réécriture de Plutarque trouve une sorte de point culminant dans l'expression célèbre «il se trouve plus de différence de tel homme à tel homme que de tel animal à tel homme $»^{30}$, qui provient de la distorsion d'une formule de Plutarque. Montaigne renvoie lui-même à une autre occurrence de cette formule, qui se trouve dans l'essai I, 42, «De l'inégalité qui est entre nous »:

Plutarque dit en quelque lieu qu'il ne trouve point si grande distance de beste à beste, comme il trouve d'homme à homme. Il parle de la suffisance de l'ame et qualitez internes. A la verité, je trouve si loing d'Epaminundas, comme je l'imagine, jusques à tel que je connois, je dy capable de sens commun, que j'encherirois volontiers sur Plutarque; et dirois qu'il y a plus de distance de tel à tel homme qu'il n'y a de tel homme à telle beste ${ }^{31}$.

Effectivement Plutarque écrit, à la fin du traité Que les animaux usent de raison :

Je ne pense pas qu'il ait si grande distance de bête à bête, comme il y a de grand intervalle d'homme à homme en matière de prudence, de discours de raison, et de mémoire ${ }^{32}$.

L'enjeu de la phrase de Plutarque est défini par les lignes qui la précèdent dans son traité : s'il y a des bêtes plus stupides que d'autres, c'est bien (à l'encontre de ce que disent les Stoïciens) que toutes les bêtes participent à l'intelligence. Le «renchérissement» de Montaigne donne sans doute un tout autre sens au texte. Il apparaît cependant à cet endroit encore assez inoffensif. On chercherait en vain une idée originale dans l'hyperbole de l'essai «De l'inégalité qui est entre nous ». Ce que Montaigne souligne ici n'est pas la faible distance qui sépare l'homme de l'animal, mais la distance importante qui sépare le "grand homme » de l'« homme vulgaire », distance qui peut être comblée par l'exercice de la vertu, à laquelle il nous faut consacrer nos forces - nous ne quittons guère ici une rhétorique assez classique de l'exhortation (l'homme vulgaire se rabaisse au rang de l'animal, seul l'homme vertueux est homme en acte, etc.). C'est là rejoindre indirectement le traité de Plutarque qui, s'appuyant sur le mythe de Circé, mettait en scène un cochon faisant la morale aux hommes. Il en va tout autrement pour le texte de l' Apologie: ce n'est pas la grande distance entre l'homme vertueux et l'homme vulgaire qui provoque l'hyperbole, mais la faible distance entre le meilleur des animaux (l'éléphant) et le commun des hommes. Il y a bien là, sous-jacente à l'expression de Montaigne, une mise en doute de la clôture générique et spécifique au profit d'une valorisation de l'individu singulier.

C'est à un désassemblage de la structure générico-spécifique, conduisant à une véritable destructuration de l'être que nous assistons dans cette utilisation des fables animales des Anciens. La conclusion sera encore une fois reprise à Plutarque : "nous n'avons aucune communication à l'estre ${ }^{33}$. Mais là où Plutarque ne faisait que reprendre un lieu commun du parménidisme et du platonisme (l'opposition de ce qui est éternellement et ce qui, devenant toujours autre qu'il n'est, n'est jamais au sens authentique du terme) ${ }^{34}$, Montaigne voit là l'expression d'une véritable expérience du chaos, traduite en une expérience à la fois métaphysique et morale. 
Soyons plus précis. Nous ne croyons pas que Montaigne ait jamais douté qu'un chien était un chien et un chat un chat ... ou un homme un homme. Nous ne croyons pas non plus qu'il ait douté qu'il y avait bien de la différence entre l'homme et les animaux. L'erreur à notre sens de ceux qui, comme Antoine Compagnon (voire Michael Screech), sur la foi de quelque phrases de l'essai « Des noms », rattachent Montaigne au nominalisme d'Ockham ${ }^{35}$ est de ne pas tenir compte de que celui-ci ne parle jamais en théoricien de la connaissance, ni en philosophe du langage ou de la nature, ni même en théologien ou en métaphysicien. Il est certain que les Essais, compris comme essais du jugement, ont la prétention de s'immiscer dans tous les domaines - il croisent ainsi les intérêts du métaphysicien, du théologien ou du théoricien de la connaissance. Il parle avant tout, voire exclusivement, en moraliste. L'objet des Essais reste cependant premièrement moral. Au projet moral se trouve subordonné le propos sceptique lui-même ${ }^{36}$. Voici comment Montaigne introduit l'ensemble de son développement sceptique de l'Apologie de Raimond Sebond:

Le moyen que je prens pour rabatre cette frenaisie et qui me semble le plus propre, c'est de froisser et fouler aux pieds l'orgueil et humaine fierté ; leur faire sentir l'inanité, la vanité et deneantise de l'homme ; leur arracher des poings les chetives armes de leur raison ; leur faire baisser la teste et mordre la terre soubs l'authorité et reverance de la majesté divine ${ }^{37}$.

A qui s'adresse ce texte? Aux seuls adversaires de Sebond, ces théologiens qui disent que ses arguments "sont foibles et ineptes à vérifier ce qu'il veut, en entreprennent de les choquer aysément ${ }^{38}$ - disons pour simplifier des théologiens "professionnels " et universitaires, opposés à la théologie laïque et "grand public » du médecin catalan? A tous ceux qui, sous prétexte de certitudes religieuses, mettent en péril la paix sociale? Aux dogmatistes en général, qui revendiquent la légitimité d'un discours résolutif sur l'être des choses et sur Dieu (et Sebond lui-même risque bien d'être compris parmi eux)? Ou est-il tourné plus généralement encore contre une attitude naturelle et universelle de l'esprit humain? Il est significatif que nous trouvions l'écho de ce texte à l'intérieur d'un essai dont l'objet est explicitement moral, l'essai II, 17, «De la praesomption», qui reprend la même terminologie :

De toutes les opinions que l'ancienneté a eües de l'homme en gros, celles que j'embrasse le plus volontiers et auxquelles je m'attache le plus, ce sont celles qui nous méprisent, avilissent et anéantissent le plus. La philosophie ne me semble jamais avoir si beau jeu que quand elle combat nostre presomption et vanité, quand elle reconnoit de bonne foy son irresolution, sa foiblesse et son ignorance ${ }^{39}$.

Le scepticisme apparaît ici comme une épreuve d'humilité. Quelle est la valeur de cette humiliation? Elle n'est certes pas un but en soi. Elle n'a pas non plus pour fin de nous faire reconnaître une autorité transcendante, plus haute que nous-mêmes. Le discours théologique n'est sans doute pas rejeté par Montaigne. Mais il ne constitue en aucun cas le projet fondamental auquel le scepticisme serait subordonné ${ }^{40}$. Le projet de Montaigne n'est ni celui de Sénèque (s'élever au dessus de l'humanité) ni celui de Plutarque (nous faire reconnaître, à travers l'expérience du néant du monde temporel, la transcendance du dieu qui seul «est » éternellement). Loin que l'expérience du néant que porte à son terme le scepticisme montaigniste nous renvoie à une dimension de l'hétéronomie ${ }^{41}$, elle nous renvoie à un projet d'immanence. Il s'agit d'écarter de notre champ de connaissance et d'action tout ce qui n'est pas nous, et qui, par conséquent, n'est pas non plus à notre portée. Ce qui n'est pas à notre portée, c'est l'être, avec lequel nous n'avons aucune communication. L'être, c'est-à-dire le monde, l'univers et son échelle prétendue, Dieu, 
nous-mêmes aussi en tant qu'êtres dans le monde - composés d'âme et de corps (l'«Apologie de Raimond Sebond» ne manque pas de critiquer toute psychologie et somatologie dogmatiques). Cette " kénose ", pour ainsi la nommer, est une forme d'épokhè morale et une pratique du retour à soi court-circuitant toute ontologie. On pourra comparer le texte de l'essai «De la praesomption» à cette phrase de l'essai «De l'experience » :

Des opinions de la philosophie, j'embrasse plus volontiers celles qui sont les plus solides, c'est à dire les plus humaines et nostres: mes discours sont, conforméement

à mes meurs, bas et humbles ${ }^{42}$.

Les opinions sceptiques qui combattant notre présomption et vanité «nous méprisent, avilissent et anéantissent» sont donc aussi les opinions qui sont «les plus humaines et nostres». La vacuité du point de vue "ontologique» fait signe vers une consistance morale du point de vue de l'immanence.

\section{Conciliatio et naturalisme moral : Montaigne face aux stoïciens et aux épicuriens}

41 Nous pourrions penser que nous avons quitté le thème de l'animalité. En réalité, celui-ci réintervient à cet endroit pour prendre une fonction morale. L'animalité ici désigne un certain mode d'appropriation à soi-même, et peut, de façon analogique tout du moins, fournir un modèle pour l'homme. L'animalité est bien loin de signifier chez Montaigne ce qu'elle signifie pour de certains éthologues modernes comme Jacob von Uexküll ou certains philosophes comme Heidegger, Derrida et Agamben - une modalité d'accaparement et de captation par le milieu ambiant ${ }^{43}$. Elle signifie au contraire pour Montaigne ce qu'elle signifie pour les stoïciens et les épicuriens - une modalité d'être à soi-même, une oikéiôsis, c'est-à-dire une façon de s'approprier à soi-même et aux autres - en ce sens, l'animalité fournit bien un modèle positif de moralité pour l'homme. Il y a cependant une différence fondamentale entre l'oikéiôsis animale et l'oikéiôsis humaine. Montaigne oscille pour ce qui est de savoir si les animaux sont mus par une forme de contrainte naturelle ou d'immédiateté d'instinct. A supposer que ce soit le cas, cette immédiateté vaut comme avantage en faveur de l'animal, qui échappe ainsi aux maux qui pressent l'homme, et qui, dans l'« Apologie ", se nomment " peché, maladie, irresolution, trouble, desespoir ${ }^{44} »$. Mais ce qui importe, c'est que l'animal ainsi compris ne saurait tracer pour l'homme qu'un modèle négatif. Car, entre cette nature à laquelle l'animal se trouve attaché et cette autre que Montaigne nous demande, à nous hommes, de suivre (en prenant comme guide non plus l'animal comme tel, mais Socrate), il y a bien de la différence. Contrairement aux morales stoïciennes ou épicuriennes, la morale montaigniste n'est pas naturaliste au sens traditionnel du terme.

Montaigne connaît et, à l'occasion, se réfère au thème stoïcien de l'oikéiôsis, sous la forme de la conciliatio cicéronienne (De finibus, I, III et V) et sénéquienne (Lettre 121 à Lucilius). Il reste que cette conciliatio ne fonde pas pour lui les rapports, même naturels, de l'homme à autrui. Lorsqu'il parle du respect dû à l'animal, par exemple, Montaigne se réfère directement au thème théophrastien de l'oikéiôtès dont il trouve quelque trace chez Plutarque et Porphyre, et qui, justement constitue un modèle alternatif à celui, stoïcien, de l'oikéiôsis. C'est le cas, par exemple, dans l'essai II, 11, « De la cruauté » :

[Il y a] un certain respect qui nous attache, et un general devoir d'humanité, non aux bestes seulement qui ont vie et sentiment, mais aux arbres mesmes et aux 
plantes. Nous devons la justice aux hommes, et la grâce et la bénignité aux autres créatures qui en peuvent être capables. Il y a quelque commerce entre elles et nous, et quelque obligation mutuelle ${ }^{45}$.

Le fondement de cet altruisme "naturel» (dont par ailleurs Montaigne doute qu'il possède chez l'homme une effectivité réelle) se trouve non dans l'extension d'un attachement primordial à soi, mais plutôt dans la solidarité des êtres, liés par une chaîne de besoins et de relations réciproques, impliquant en retour une chaîne de devoirs mutuels. D'où l'extension possible (et impensable pour les stoïciens) de nos devoirs aux bêtes et même aux plantes.

Il est plus important de souligner le fait que la moralité n'est jamais pour Montaigne une simple extension de la conciliatio naturelle, mais qu'elle implique une rupture. Une rupture de fait tout d'abord: si la présence d'une forme d'attachement naturel à soi et à l'autre est indiscutable chez les bêtes, elle est par contre très incertaine en l'homme qui reste toujours pour Montaigne un animal dénaturé. Il y a des exceptions, tels les cannibales de l'essai I, 31 ou les sauvages du nouveau monde de l'essai III, 6, qui continuent à vivre gouvernés par les lois naturelles non corrompues par la science. Il y a aussi les modèles de vertu du passé (Épaminondas en étant le meilleur exemple, Étienne de la Boétie en étant peut-être le vestige unique dans les temps modernes), pour qui la nature passe en vertu comme par une sorte de continuité naturelle. Tous ces portrait ont une fonction très précise dans les Essais: ils dessinent en creux le nôtre : ils accusent ce que nous ne sommes pas, ne savons pas être et ne pouvons plus être, nous, hommes de ce siècle, corrompus par la science et l'hypertrophie de la raison (nous aussi, lecteurs auxquels s'adressent les Essais), sinon peut-être de façon fortuite - car il arrive que la loi naturelle reprenne le dessus, sous la forme d'inclinaison d'affection (par exemple la pitié qui nous empêche d'égorger sans quelque réticence l'animal pris à la chasse) alors que nous ne nous y attendons pas.

Mais la rupture est aussi de droit. Nous voulons dire qu'elle est pleinement légitimée pour Montaigne. Comme Carnéade et Cicéron (contre les stoïciens), Montaigne voit dans la raison une véritable rupture vis-à-vis de l'ordre de la nature. Lorsque Montaigne parle de la loi naturelle dans l'essai II, 8, «De l'affection des pères aux enfants », c'est dans un sens restrictif :

S'il y a quelque loy vrayement naturelle, c'est à dire quelque instinct qui se voye universellement et perpétuellement empreinct aux bestes et en nous (ce qui n'est pas sans controverse), je puis dire, à mon avis, qu'après le soing que chasque animal a de sa conservation et de fuir ce qui nuit, l'affection que l'engendrant porte à son engeance, tient le second lieu en ce rang ${ }^{46}$

On aura remarqué ici la forme modale de la phrase montaigniste ( $S$ ' $i l$ y a quelque loi vrayement naturelle », « ce qui n'est pas sans controverse »). Il n'est encore une fois pas certain que l'homme soit encore gouverné par une «nature », comme l'est l'animal. Mais peut-être d'ailleurs que ce qui est sujet à controverse est le gouvernement par la nature à la fois de l'homme et de l'animal - au fond, ce n'est pas là l'essentiel, et c'est peut-être là la clé de la contradiction de notre second texte. Ce qui est important, c'est le caractère restrictif et limité de cette conciliatio. L'affection que nous portons à notre progéniture ne prend une valeur « morale » que lorsqu'elle se fonde non plus sur un sentiment de nature, mais sur une estime raisonnable - c'est là tout le sens de cet essai « De l'affection des pères aux enfants $»$. 
ce rupture affecte non seulement nos rapports avec autrui, mais aussi et surtout notre rapport à nous-même, ou encore ce que Montaigne nomme à un endroit cette "amitié que chacun se doibt $"{ }^{47}$. Nous nous trouvons en réalité en face de deux figures morales de l'animalité. La première désigne un attachement naturel et immédiat à soi-même et à autrui - elle ne constitue pour l'homme qu'un modèle inaccessible et même négatif et a un rôle moral essentiellement parénétique. La seconde consiste dans un attachement à soi et aux autres reconstruit sur la base de la raison à l'intérieur même de l'immanence de l'homme: cette animalité est construite, elle est d'une certaine façon fictive, elle est pleinement anthropomorphique (même si cet anthropomorphisme ne vise plus la moindre domination herméneutique sur le monde). C'est à ce prix que l'animalité devient un modèle positif pour l'homme.

mieux comprendre ce point, citons un texte tiré de l'essai « De la physionomie ». Dans cet essai, Montaigne tente de définir ce que pourrait être un juste rapport de l'homme à la nature. De ce juste rapport, les animaux nous montrent quelque exemple. Mais quel exemple exactement? Tout d'abord celui d'une soumission à la nature qui les exempte de toutes les inquiétudes dont les hommes sont victimes :

Nature a emprunt aux bestes le soing d'elles et de leur conservation. Elles vont jusques là de craindre leur empirement, de se heurter et blesser, que nous les enchevestrons et battons, accidents subjects à leurs sens et experience. Mais que nous les tuons, elle ne le peuvent craindre, ny n'ont la faculté d'imaginer et conclurre la mort ${ }^{48}$

La matière de ce texte est stoïcienne. Mais Montaigne insiste ici sur le caractère limité de conciliatio stoïcienne, même dans le strict cadre de l'attachement à soi-même: celle-ci n'inclut que la préservation des maux présents, non l'anticipation des maux à venir. On trouvait ce thème déjà présent chez Sénèque. Mais celui-ci s'attachait à montrer que la même conciliatio, limitée chez l'animal aux biens de conservation présents, était chez l'homme étendue aux biens raisonnables (se conserver, mais comme être de raison - donc par prévision de l'avenir et anticipation de la mort). Montaigne insiste ici au contraire sur la rupture opérée par la raison. Sans doute, il serait merveilleux que les hommes eux aussi puissent vivre limités dans l'instant sans anticiper l'avenir. Mais il nous faut d'emblée renoncer à cette ignorance heureuse. La délivrance de la crainte a pour l'homme un autre prix. Ce que la nature ne nous fournit plus, c'est une tendance, une hormè. La dynamique morale part de tout autre chose que de l'impulsion instinctive. Il n'y a pas chez Montaigne d' « argument des berceaux » (pour reprendre la formule de Jacques Brunschwig), au sens d'une volonté de fonder la norme morale dans la factualité de la nature. C'est en ce sens qu'il convient de comprendre cette phrase tirée de l'essai « De l'expérience »:

La douleur, la volupté, l'amour et la haine sont les premières choses que sent un enfant ; si, la raison survenant, elles s'appliquent à elle, cela est vertu ${ }^{49}$.

C'est ici à la version épicurienne de l'oikéiôsis que Montaigne s'oppose : non qu'il ne pense que la morale ait un rapport étroit au plaisir et à la peine. Il reste qu'elle ne s'origine pas dans la naturalité du sentiment. La raison peut venir se superposer à la nature, mais elle ne la prolonge pas - elle désigne une autre origine, qui seule peut proprement qualifier un acte de moral. La raison introduit ainsi un nouveau fondement dans la moralité.

51 La nature continue cependant à nous fournir quelque chose comme un modèle moral, ne serait-il qu'analogique. La suite du texte de l'essai «De la physionomie » modifie en 
substance la première figure de l'animalité comme relation immédiate à la nature, pour en introduire une autre :

[C] Si dict-on encore qu'[B] on les voit non seulement la souffrir gayement (la plus part des chevaux hannissent en mourant, les cignes la chantent), mais de plus la rechercher à leur besoing, comme portent plusieurs exemples des elephans ${ }^{50}$.

Voici maintenant que les animaux ne vivent plus limités à l'instant dans l'ignorance de l'avenir, mais au contraire anticipent (joyeusement) cet avenir ${ }^{51}$. C'est le souci de trouver dans l'animal un modèle de moralité pour l'homme qui provoque l'hyperbole: non seulement les animaux n'anticipent pas la mort, mais ils l'attendent avec gaieté ou la recherchent. Il y a pour le coup une contradiction apparente entre les deux couches [C] (les animaux n'anticipent pas la mort) et [B] (les animaux aiment la mort et en jouissent). Mais la contradiction fait signe vers une cohérence plus profonde. Le cygne du Phédon déborde le modèle sénèquéen, parce que l'impréméditation est en réalité pour l'homme (auquel ici l'animal s'adresse) le fruit d'une préméditation, c'est-à-dire d'un travail moral. Ne pas penser à la mort, trouver des moyens pour divertir l'esprit de sa pensée, ce n'est en réalité qu'y penser toujours sur un certain mode. A cet endroit, la figure de l'animal rejoint tout simplement celle de Socrate pour définir ce que peut être le juste rapport de l'homme à la nature.

L'homme n'a pas d'animalité immédiate. Cette animalité est le fruit pour lui d'une construction. Citons un texte tiré de l'essai « De la solitude »:

Il faut faire comme les animaux qui effacent la trace, à la porte de la taniere [...]. Retirez-vous d'abord en vous, mais preparez vous premierement de vous y recevoir: ce seroit folie de vous fier à vous mesmes, si vous ne vous sçavez gouverner (I, 39, 247 [A]).

La référence animale n'est ici qu'analogique. Il faut à l'homme construire sa tanière, et construire sa tanière, c'est pour lui c'est se savoir gouverner selon la raison - donc sur un mode humain. Trouver le juste rapport à ce corps qui nous sert de mesure suprême (l' ariston métron, à la fin de l'essai «De l'experience ${ }^{52}$ ) dans notre absence de rapport à toute mesure dans l'être.

Terminons en insistant sur cette place du corps animal dans l'économie de la philosophie morale de Montaigne. Il arrive à Montaigne de prendre la défense des animaux contre les mauvais traitements infligés par l'homme. Non que Montaigne interdise la consommation carnée ou condamne la chasse, selon la perspective du De abstinentia de Porphyre, ou du traité de Plutarque S'il est loisible de manger de la chair (mais aussi de la première partie du traité Quels animaux sont les plus avisés ?). Il est vrai que l'essai II, 12, « De la cruauté » qui précède l'«Apologie de Raimond Sebond", condamne les mauvais traitements infligés aux animaux. Ce n'est pas cependant là l'objet direct du propos moral de Montaigne, et ce pour deux raisons : 1 / Parce que tout d'abord la critique des mauvais traitements infligés aux animaux n'est qu'un cas particulier (et peut-être particulièrement significatif) d'une critique plus générale de la prédation herméneutique opérée par l'homme sur le monde et l'être. Nous voulons dire que ce thème de la défense des animaux ne constitue pas chez Montaigne une thématique spécifique, comme c'est le cas chez Plutarque ou Porphyre. 2 / Parce que Montaigne ne fait là que développer un des arguments de Plutarque, selon qui ces mauvais traitements se retournent contre l'homme lui-même: contre ses semblables tout d'abord, car la cruauté est bien pour Montaigne le plus haïssable de tous les maux et l'extrême de tous les vices ${ }^{53}$. Mais pour Montaigne, qui ici innove totalement par rapport à Plutarque (et plus généralement par rapport à toute anthropologie de type 
platonicien), il y a aussi une forme de cruauté de l'homme contre lui-même sous la forme d'un ressentiment contre le corps. L'homme est ce «monstrueux animal qui se fait horreur à soy mesme, à qui ses plaisirs poisent ; qui se tient à mal-heur " ${ }^{54}$.

L'appropriation à soi-même passera ainsi par une appropriation de son corps animal, qui n'est pas, comme l'ont cru certains rationalistes intempérants ou dualistes platonisants, source d'hubris, mais au contraire source de stabilité et de rationalité face aux divagations de l'esprit laissé à lui-même: «je trouve ma concupiscence moins desbauchée que ma raison ", écrit Montaigne dans cet essai « De la cruauté " ${ }^{55}$.Le thème trouvera toute son ampleur dans l'essai « De la praesumption»:

Le corps a une grand'part à nostre estre, il y tient un grand rang [...]. Ceux qui veulent desprendre nos deux pieces principales et les sequestrer l'une de l'autre, ils ont tort. Au rebours, il les faut r'accoupler et rejoindre. Il faut ordonner à l'ame non de se tirer à quartier, de s'entretenir à part, de mespriser et abandonner le corps (aussi ne le sçaurait elle faire que par quelque singerie contrefaicte), mais de se r'allier à luy, de l'embrasser, le cherir, luy assister, le contreroller, le conseiller, le redresser et ramener quand il fourvoye, l'espouser en somme et luy servir de mary 56.

Le corps est pour Montaigne principe immanent de mesure, à même de servir de norme pour une morale privée par avance de toute relation à l'être des choses. Nous passons bien ici du thème de l'intelligence et de la vertu des animaux à celui, plus essentiel dans la pensée de Montaigne de l'intelligence et de la vertu de l'animalité. Il y a là, si l'on veut, un thème épicurien: il reste que si le corps désigne sans doute chez Montaigne une immédiateté, la relation au corps, elle, n'est pas immédiate. Encore une fois, ce qui était pour les épicuriens le fruit d'une morale naturelle fondée sur l'oikéiôsis devient pour Montaigne le fruit d'une morale construite, qui ne veut pas ignorer la part proprement rationnelle de l'homme ${ }^{57}$.

\section{NOTES}

1. Il faut ajouter à cela les références moins nombreuses au traité « Que les bêtes brutes usent de raison ». Sur le rapport de Montaigne à Plutarque, nous renvoyons le lecteur à notre ouvrage De l'homme à l'animal. Montaigne, Descartes ou les paradoxes de la philosophie moderne sur la nature des animaux, Paris, Vrin, 1998, p. 69-84.

2. Il est cependant vrai que Montaigne ne pouvait guère connaître l'Alexander, qui constitue aujourd'hui une source très précieuse, en en tout cas la plus ancienne, sur cette question. Cf. à ce sujet notre ouvrage, L'Homme et l'animal. La philosophie antique, Paris, PUF, 1999, p. 88 sq.

3. . Nous avons fourni un plan détaillé de ce texte (et même deux, selon deux perspectives différentes) dans notre ouvrage De l'homme à l'animal ..., éd. cit., p. 42-43.

4. . Académiques, seconde version, I, 12, 45 dans Cicero, De natura deorum - Academica, trad. angl. H. Rackham, Cambridge-London, Harvard University Press, 1933, rééd. 2000 [éd. Rackham], p. 452.

5. . Cicéron, Académiques, première version, II, XXV, §80-81, trad. E. Bréhier, dans Les Stoïciens, Paris, Tel Gallimard, 1962, t. I, p. 224 (éd. Rackham, p. 568). Nous soulignons.

6. . Platon, Théétète, 154 a. 
7. . Ibid., $161 \mathrm{c}$.

8. . Toutes nos références latines au texte de Sextus sont tirées de l'édition d'Henri Estienne, Sexti philosophi Pyrrhoniarum hypotypôséôn libri III, Paris, 1562 [éd. H. Estienne] (ici p. 18).

9. . Sextus Empiricus, Hypotyposes pyrroniennes, I, XIV, 61, éd. H. Estienne, p. 23.

10. . Ibid., I, XIV, 40, éd. H. Estienne, p. 18.

11. . Essais, II, 12, éd. P. Villey, p. 587.

12. . Montaigne, Essais, II, 12, 587-588 [A].

13. . Essais, II, 12, 597 [A].

14. . Essais, II, 12, 452 [A].

15. . Essais, II, 12, 467 [A]. Nous avons tenté d'éclairer ce texte complexe dans une étude «Sur quelques "contradictions" de la zoologie de Montaigne", que nous espérons voir paraître prochainement.

16. . Essais, II, 12, 532-533 [B].

17. . Essais, II, 12, 452 [C].

18. . Essais, II, 12, 452 [A].

19. . Cf. sur ce point C. Levy, Cicero Academicus. Recherches sur les Académiques et sur la philosophie cicéronienne, Collection de l'École Française de Rome, 1992, p. 218-223.

20. . Académiques, $1^{\mathrm{e}}$ version, II, VII, 19, éd. Rackham, p. 492, trad. E. Bréhier, dans op. cit., p. 197.

21. . Quels animaux sont les plus avisés ..., éd. E. de Fontenay, P.O.L., 1992 [éd. Fontenay], p. 191 (Les Cuvres morales et philosophiques, trad. J. Amyot, Paris, 1572 [éd. Amyot], fol. 517 G). On retrouve une prudence semblable (elle aussi rejetée par Montaigne) dans les exemples qu'Augustin donne de corps capables de chauffer ou de brûler sans se corrompre en Cité de Dieu, XXI, 5 : cf. à ce sujet notre article «L'essai et l'expérience : le scepticisme montaigniste au-delà du fidéisme ", dans M.L. Demonet et A. Legros (dir.), L'Écriture du scepticisme chez Montaigne, Genève, Droz, 2004, p. 226-227.

22. . Quels animaux sont les plus avisés ..., éd. Fontenay, p. 196 (éd. Amyot, fol. 519 A).

23. Quels animaux sont les plus avisés ..., éd. Fontenay, p. 214 (éd. Amyot, fol. 523 C).

24. . Quels animaux sont les plus avisés ..., éd. Fontenay, p. 216 (éd. Amyot, fol. $523 \mathrm{G}$ ).

25. . Quels animaux sont les plus avisés..., éd. Fontenay, p. 175 (éd. Amyot, fol. 513 H). Nous soulignons.

26. . Sextus Empiricus, Hypotyposes pyrhoniennes, I, 14, 69, éd. H. Estienne, p. $24-25$ (nous utilisons ici la traduction de Pierre Pellegrin chez GF Flammarion, p. 93).

27. . Essais, II, 12, 463 [A].

28. . Essais, II, 12, 460 [A].

29. . Une autre illustration pourrait être donnée à partir de la reprise de l'histoire du rémora. Nous renvoyons le lecteur à notre ouvrage De l'homme à l'animal ..., op. cit., p. 75-76.

30. . Essais, II, 12, 466 [A].

31. Essais, I, 42, 258 [A].

32. . Que les animaux usent de raison, éd. Amyot, fol. $274 \mathrm{~A}$.

33. . Essais, II, 12601 [A].

34. . Cf. Parménide, Poème, VIII, 5 sq. ainsi que Platon, Cratyle, 439 e et Timée 27 d-28 a.

35. . A. Compagnon, Nous, Michel de Montaigne, Paris, Le Seuil, 1980, p. 53-142.

36. . Nous avons développé ce point dans notre article «Pourquoi l'homme n'est-il plus un animal raisonnable? Montaigne, Descartes, ou les raisons d'un refus », dans Th. Gontier (dir.), Animal et animalité dans la philosophie de la Renaissance et de l'Âge Classique, Louvain-la-Neuve, Peeters, 2005, en particulier p. 117-121.

37. . Essais, II, 12, 448 [A].

38. . Essais, II, 12, 448 [B]).

39. . Essais, II, 17, « De la praesumption », 634 [A et C]. 
40. . Sur le rapport de Montaigne au "fidéïsme" de son époque, cf. notre article "L'essai et l'expérience : le scepticisme montaigniste au-delà du fidéisme ", art. cit.

41. . Cf. notre article « Finitude du Dasein, finité humaniste », B. Pinchard, Heidegger et la question de l'humanisme. Faits, concepts, débats, Paris, PUF, p. 13-33.

42. . Essais, III, 13 « De l'experience », 1113 [B].

43. . Thème abordé en conclusion de notre étude « « Pourquoi l'homme n'est-il plus un animal raisonnable ? ... », art. cit., p. 127-128.

44. . Essais, II, 12, 460 [A].

45. . Essais, II, 11, « De la cruauté », 435 [A].

46. . Essais, II, 8, « De l'affection des pères aux enfants », 386 [B]

47. . Essais, III, 10, «De mesnager sa volonté », 1006 [B]). L'amitié est pour Montaigne ce qui donne tout le prix à la raison humaine - et cette amitié, les animaux incontestablement l'ignorent, car elle est fondée sur un acte radicalement libre et délié de toute contrainte naturelle - on se réfèrera sur ce point à l'essai I, 28, « De l'amitié ».

48. . Essais, III, 12, 1055 [C].

49. . Essais, III, 13, 1111 [C].

50. . Essais, III, 12, 1055 [B et C].

51. . La matière de ce texte est stoïcienne. Mais Montaigne insiste ici sur le caractère limité de conciliatio stoïcienne, même dans le strict cadre de l'attachement à soi-même: celle-ci n'inclut que la préservation des maux présents, non l'anticipation des maux à venir. On trouvait ce thème déjà présent chez Sénèque. Mais celui-ci s'attachait à montrer que la même conciliatio, limitée chez l'animal aux biens de conservation présents, était chez l'homme étendue aux biens raisonnables (se conserver, mais comme être de raison - donc par prévision de l'avenir et anticipation de la mort). Montaigne insiste ici au contraire sur la rupture opérée par la raison.

52. . « Moy, qui ay tant adoré, et si universellement, cet ariston metron du temps passé et ay pris pour la plus parfaicte la moyenne mesure, pretendray-je une desmesurée et monstrueuse vieillesse? » (Essais, III, 13, p. 1102 [C]).

53. . Essais, II, 11, 429 [B].

54. . Essais, III, 5, « Sur des vers de Virgile, 879 [B et C].

55. . Essais, II, 11, 428 [B].

56. . Essais, II, 17, « De la praesumption, 639 [A].

57. Cet article est issu d'un exposé fait dans le cadre du séminaire du LALIA à Nice le 28 avril 2005.Nous remercions Arnaud Zucker de nous avoir permis de reprendre selon une perspective nouvelle des thèmes développés dans notre thèse de 1996.

\section{RÉSUMÉS}

Le discours des Essais de Montaigne se nourrit de références constantes à la littérature zoologique antique. Nous voulons analyser ici les modalités de la translatio de certaines de ces références, à partir de la confrontation de Montaigne (1) à l'argumentation de Sextus Empiricus, et plus généralement à la littérature anti-stoïcienne, (2) aux mirabilia de Plutarque, (3) à la morale naturaliste, fondée sur la notion d'oïkéiosis ou de conciliatio, des stoïciens et des épicuriens. Cette triple confrontation nous permet de comprendre l'articulation centrale chez Montaigne du propos sceptique à un propos moral. L'animalité revêt ainsi une double fonction : à 
l'intérieur $\mathrm{du}$ discours sceptique, elle est utilisée dans une critique générale de l'anthropocentrisme, soit d'un certain rapport de l'homme à l'être, au monde et à lui-même ; à l'intérieur du discours moral, elle définit un modèle analogique et conduit à envisager le caractère éthique de la relation de l'homme à son corps.

The Montaigne's Essays constantly refers to the antic zoological literature. In this paper we analyze the translatio of some of those references, considering the relation of Montaigne to (1) Sextus Empiricus' argumentation (and the anti-Stoic literature in general), (2) Plutarch's mirabilia, and (3) the naturalistic morality, based on the Stoic and Epicurean notion of oikéiosis or conciliatio. This threefold confrontation enables us to appreciate the crucial connection, in the works of Montaigne, between sceptic and moral concerns. The bestiality is indeed playing two different roles: in the sceptic discourse, it takes place in a general criticism of anthropocentrism, i.e. of the special relation man has to being, world, and himself ; in the moral speech, it defines an analogical model and leads to take in account the ethical aspect of the relation of human being to his own body.

INDEX

Mots-clés : Montaigne, Plutarque, Sextus Empiricus, stoïciens, zoologie

\section{AUTEUR}

\section{THIERRY GONTIER}

Professeur de philosophie à l'Université de Lyon III 